\title{
Research on Geometric and Kinematic Analyses of Some Auxiliary Mechanisms Used on Urban Buses
}

\author{
Daniela Antonescu ${ }^{1}$, Daniel Iozsa $^{2}$, Ovidiu Antonescu ${ }^{3}$ and Gh. Fratila ${ }^{4}$ \\ 1 "Iuliu Maniu" High School, Bucharest, Romania, ${ }^{2}$ Dept. of Road Vehicles, Politehnica \\ University of Bucharest, Romania; ${ }^{3}$ Dept. of Mechanism and Robot Theory, Politehnica University of \\ Bucharest;Romania; ${ }^{4}$ Dept. of Road Vehicles, Politehnica University of Bucharest, Romania \\ Corresponding author e-mail: daniela.mihalache@yahoo.com
}

Article history

Received 10.07.2017

Accepted 22.09.2017

DOI https://doi.org/10.26825/bup.ar.2017.003

\begin{abstract}
The paper presents the geometric-kinematic modeling of an articulated planar mechanism used to actuate the urban bus doors. The stages of solving the kinematic parameters as displacement, velocity and acceleration of each component of the linkage are performed. One of the goals of the paper is to use this modeling of kinematics to improve the operation of the mechanism as synchronization of the two folding doors. The velocity and acceleration of them are also important due to the fact that it can create a safe environment for the passengers. Before this study, a similar type of linkage used on trolleybuses is presented in brief. Also, a review of the main types of urban bus doors is emphasized in section 2 .
\end{abstract}

\section{Introduction}

For a high frequency of stops and a high number of passengers getting on and off, as well as for ensuring the safety of the passengers on boarding and during the journey, the urban vehicles are equipped with doors consisting of two or more folding parts, pneumatically or electrically controlled.

A mechanism for actuating the city bus doors is generally composed $[1,17]$ of two main parts: a) the control mechanism, mounted either under the stairs or above the door; b) the crank-slide final mechanisms to which either the crank and the coupler or only the couplers or the cranks are rigidly linked to one part of the folding door. 
The structural and kinematic analysis of the pneumatic mechanisms used to actuate the urban bus doors [4] highlights the unitary character of the control mechanisms.

Also, a general method of structural and geometric analysis of the control mechanisms, in openclosed positions, as well middle positions, is of interest.

In section 2 of the paper the main types of urban bus doors are presented. The most used mechanisms for actuating the doors are with circular sliding or rotational-sliding motions.

In section 3 a linkage having only revolute joints in the final mechanism structure, used on trolleybuses, is presented.

Finally, in section 4, a geometric-kinematic modeling of a two folding door-part mechanism is performed. The displacement, velocity and acceleration of each element of the linkage have been calculated and displayed. Based on these results, some improvements regarding the passenger comfort are outlined.

\section{Mechanisms for bus doors}

\subsection{Swinging door (conventional) with hinge joint}

In the case of classical design buses (Figure 1a) the doors have a swinging movement around a vertical axis by means of some hinges.

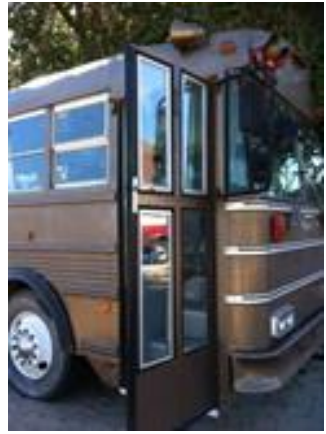

a)

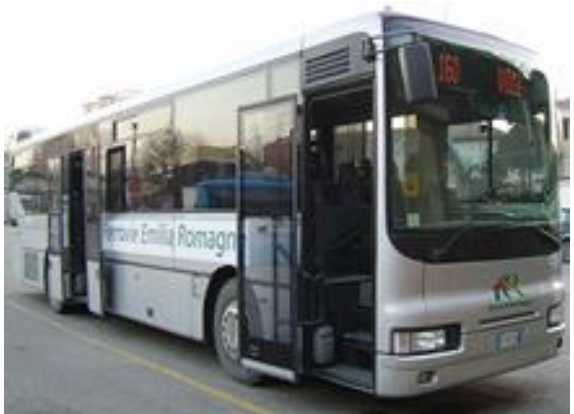

b)

Figure 1. Circular sliding doors $a$ ) and circular sliding doors (b) [18]

\subsection{Door with circular sliding motion}

In modern buses one of the door movement solutions is circular sliding, being done by means of an articulated parallelogram mechanism (Figure 1b). The kinematic scheme of such a linkage shows that the vehicle door (represented by the segment $\mathrm{MN}$ ) is rigid connected with the coupler $\mathrm{AB}$ (Figure 2).

In the practical case of bus doors, the positioning of the fixed joints $\mathrm{A}_{0}$ and $\mathrm{B}_{0}$ is made inside the body 0 , in the area of the stairway.

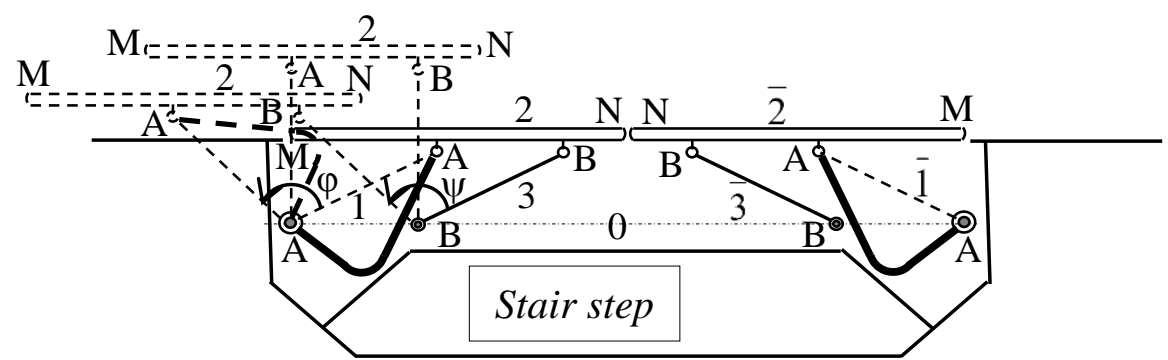

Figure 2. The kinematic scheme of the double symmetrical parallelogram mechanism

Each of the two door-parts is rigid with the coupler 2 of the parallelogram mechanism $A_{0} A B B_{0}$ in the closed position of the door with width $M N$. In the open position of the door $M^{\prime \prime} N^{\prime \prime}$, the parallelogram mechanism is $A_{0} A^{\prime \prime} B^{\prime \prime} B_{0}$. Note that the bar 1 cannot be in a straight line as it would 
interfere with the bus body, so that the shape of it is curved and it does not collide with the vehicle body (Figure 2).

The kinematic scheme of the parallelogram mechanism was drawn in three positions, two extreme positions (closed and open) and an intermediate position $M^{\prime} N^{\prime}$ at the maximum distance of the body. For each door-part a parallelogram mechanism is corresponding, whose kinematic schemes are symmetrically represented.

Due to the fact that the bar $1\left(A_{0} A\right)$ is the driving kinematic element, it has a much larger crosssection than the bar 3 , having primarily a geometric role.

Note that for the right part, the parallelogram mechanism was only in the closed position represented.

\subsection{Door with planar rotational-sliding motion}

The door of the bus can be made of two parts articulated each other, of which one of the parts performs a horizontal rotation and the other one performs a planar rotational-sliding motion.

The most commonly used solution is the one in which the door is made of a single part having a horizontal planar movement (rotational-sliding). When the door is opened, the door-part is fully folded (Figure 3a) or partially folded (Figure 3b) inside the bus.

In an intermediate position of the door opening movement (Figure 3) it is observed that the doorpart is rigid connected to the coupler of a crank-coupler planar mechanism (Figure 4).

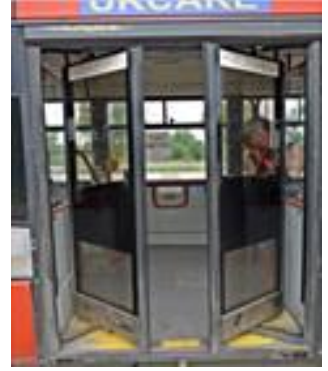

Figure 3. Doors with one-part inside folding

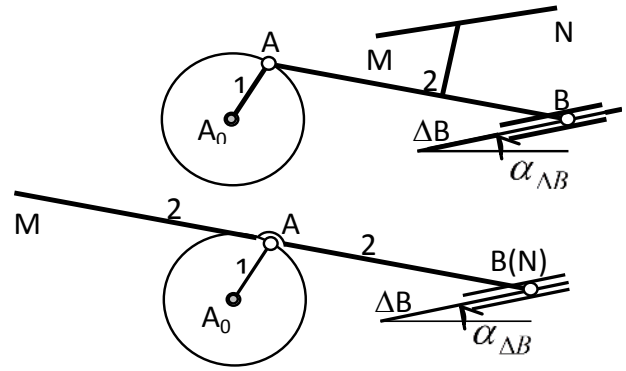

Figure 4. The kinematic scheme of the crankcoupler planar mechanism

The control and actuation mechanism of the door is usually located at the top of the bus body due to the saved space inside the bus and the lower road clearance.

\section{The door mechanism of a trolleybus type TV}

This type of mechanism (Figure 5) consists of three series-connected mechanisms: MC(1,2,3), ME1 $(3,4,5,6,7)$ and ME2 $(6,8,9)$. The main structure of the linkage is a planar triadic chain.

The final mechanism consists of a parallelogram $(5,6,7)$ and an anti-parallelogram $(6,8,9)$ so that the transmission ratio [9] achieved between the driven elements 7, 9 is -1 .

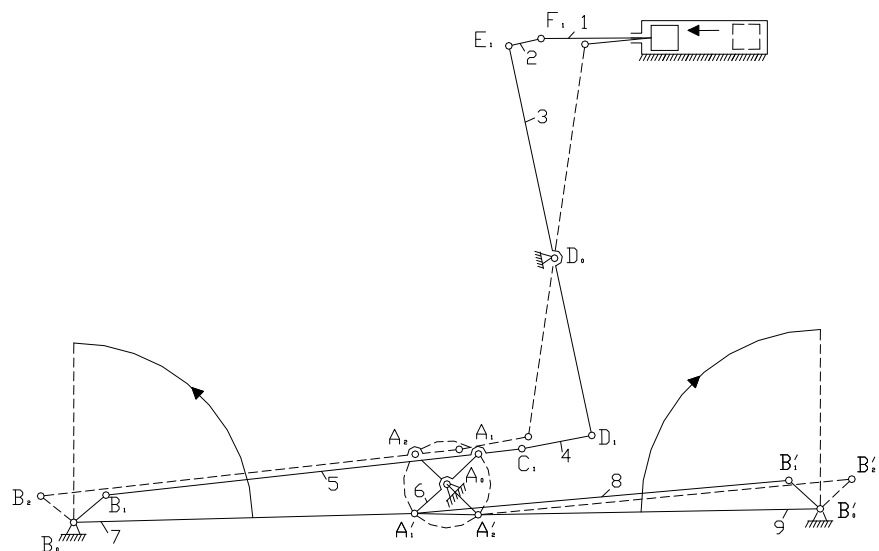

Figure 5. The kinematic scheme of planar triadic chain 
The piston 1 operates with double effect, the compressed air acting on both sides, and by means of the connecting rod 2 the linear sliding motion is turned into a rotational motion, swinging the rocker 3 (Figure 5).

Further, the movement is transmitted, by the coupler 4 , to the parallelogram $(5,6,7)$ and then, via the element 6 , to the anti-parallelogram $(6,8,9)$.

The doors or the main parts of the folding doors are fixed to the driven elements 7, 9 (having limited rotation up to $90^{\circ}$ ), according to the kinematic scheme.

\section{The geometric-kinematic modeling of a bus door mechanism}

The next kinematic scheme (Figure 6) shows the piston 1 as driving element which is positioned, at one moment in time, by means of the linear displacement $s_{10}=s_{1}$ in the fixed horizontal cylinder 0 . Through the displacement of the point $\mathrm{A}$, the two elements of the dyad chain $\operatorname{LC}(2,3)$, the coupler 2 and the rocker 3 , will incline by the angles $\varphi_{2}$ and $\varphi_{3}$ measured in the points $\mathrm{B}$ and $\mathrm{B}_{0}$.

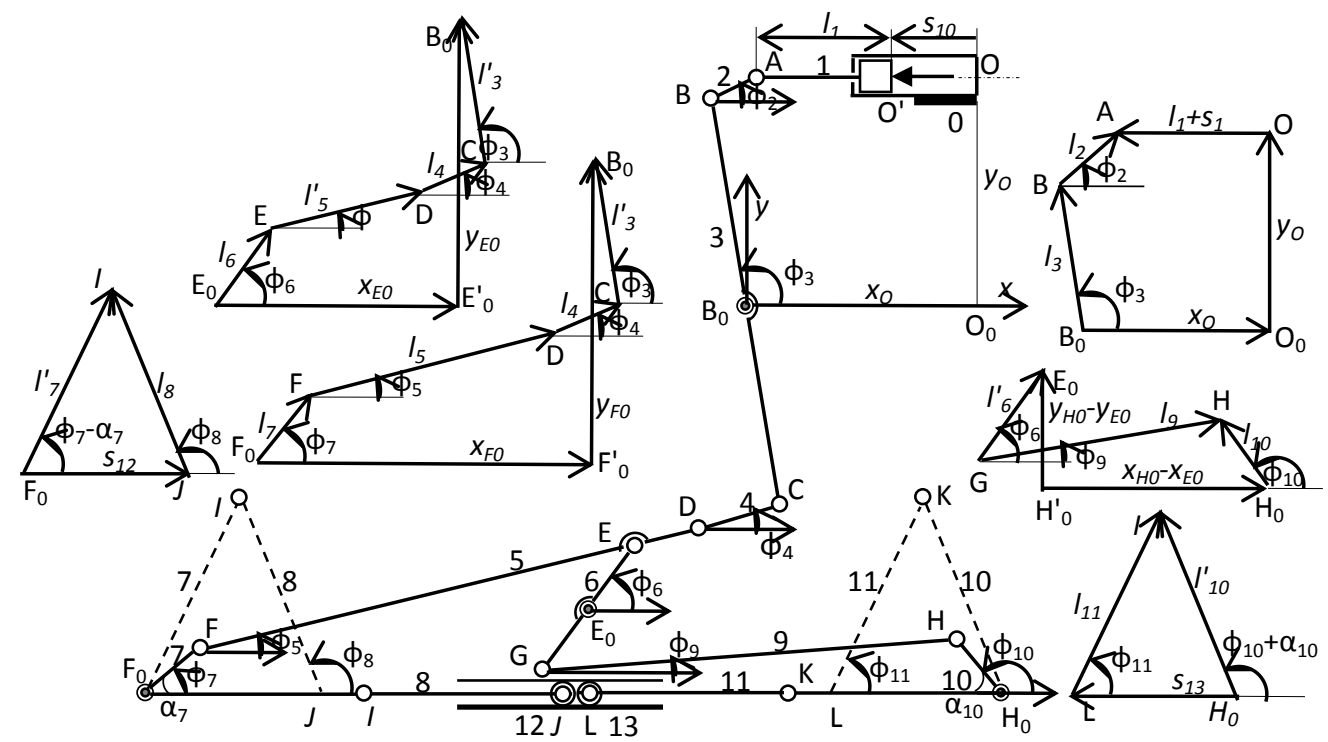

Figure 6. The kinematic scheme of the bus door mechanism (in closed position) and the independent kinematic contours

Further, the swinging movement of the point $\mathrm{C}$ is transmitted through the coupler 4 to the triadic chain $\mathrm{LT}(4,5,6,7)$ with two fixed articulations $\mathrm{E}_{0}$ and $\mathrm{F}_{0}$ (Figure 6).

The element 6 has two articulations opposed along the diameter $\mathrm{E}$ and $\mathrm{G}$, so that through the rotate joint $\mathrm{G}$ the motion is transmitted to the dyad chain $\operatorname{LC}(9,10)$.

On each of the bars 7 and 10 the first door-part is rigid connected, by which the second door-part 8 and 11 is articulated (in the points I and $\mathrm{K}$ ) whose movement is type planar rotational-sliding. The point $\mathrm{J}$ and $\mathrm{L}$ have sliding motion through the rollers 12 and 13 that move in a rectilinear guide.

Note that for the extreme displacement of the piston $1\left(s_{\text {Imax }}\right)$ the two doors are closed, the two door-parts of one door being in prolongation: $(7,8)$ and $(10,11)$.

Starting with the actuator mechanism $\mathrm{MA}(0,1)$, the structural formula of the planar "motor mechanism" $(\mathrm{MM})$ has the following expression:

$$
M M=M A(0+1)+L D(2+3)+L T(4+5+6+7)+L D(8+9)+L D(8+12)+L D(11+13)
$$

According to this formula of structural compounding of the motor mechanism, six independent closed kinematic contours have been identified, of which four contours specific to the four dyad chains and two independent contours specific to one tetrad chain.

The characteristic lengths of the linkage are relative constant parameters with respect to the coordinate system chosen in point $\mathrm{B}_{0}$, and the absolute constant parameters are fixed or mobile (the 
characteristic lengths of the kinematic elements represented by the distances between their articulation centers).

The characteristic geometric parameters with constant values are the following lengths and angles:

$$
\begin{aligned}
& x_{O}=380 \mathrm{~mm} ; y_{O}=400 \mathrm{~mm} ; x_{E_{0}}=-200 \mathrm{~mm} ; y_{E_{0}}=-390 \mathrm{~mm} ; \\
& x_{F_{0}}=-860 \mathrm{~mm} ; y_{F_{0}}=-450 \mathrm{~mm} ; x_{H_{0}}=460 \mathrm{~mm} ; y_{H_{0}}=-450 \mathrm{~mm} ; \\
& l_{1}=O^{\prime} A=240 \mathrm{~mm} ; l_{2}=A B=60 \mathrm{~mm} ; l_{3}=B B_{0}=400 \mathrm{~mm} ; l_{3}^{\prime}=B_{0} C=320 \mathrm{~mm} ; \\
& l_{4}=C D=130 \mathrm{~mm} ; l_{5}=D F=740 \mathrm{~mm} ; l_{7}=F F_{0}=90 \mathrm{~mm} ; \alpha_{7}=\angle\left(F F_{0} I\right)=45^{0} ; \\
& l_{7}^{\prime}=F_{0} I=320 \mathrm{~mm} ; l_{8}=I J=320 \mathrm{~mm} ; \\
& l_{5}^{\prime}=D E=80 \mathrm{~mm}, l_{6}=E E_{0}=90 \mathrm{~mm}, l_{6}^{\prime}=E_{0} G=90 \mathrm{~mm} ; \\
& l_{9}=G H=660 \mathrm{~mm} ; l_{10}=H H_{0}=90 \mathrm{~mm} ; \alpha_{10}=\angle\left(H H_{0} \mathrm{~K}\right)=45^{0} ; \\
& l_{10}^{\prime}=H_{0} K=320 \mathrm{~mm} ; l_{11}=K L=320 \mathrm{~mm} ;
\end{aligned}
$$

The variable lengths are the linear displacements achieved by the elements 1 (actuator piston) and the rollers 12 and 13 in the horizontal guide (Figure 6):

$$
O O^{\prime}=s_{10}=s_{1} ; F J=s_{12} ; H_{0} L=s_{13} .
$$

For each planar closed contour two scalar equations can be written, so that for the entire mechanism twelve scalar equations with twelve unknowns are deduced, that represent ten angular displacements $\varphi_{i}(i=2, \ldots, 11)$ and two linear displacements $s_{i}(i=12,13)$.

$$
\begin{aligned}
& l_{2} \cos \varphi_{2}+l_{3} \cos \varphi_{3}=x_{O}-\left(l_{1}+s_{1}\right) \\
& l_{2} \sin \varphi_{2}+l_{3} \sin \varphi_{3}=y_{O} \\
& l_{3}^{\prime} \cos \varphi_{3}+l_{4} \cos \varphi_{4}+l_{5} \cos \varphi_{5}+l_{7} \cos \varphi_{7}=-x_{F_{0}} \\
& l_{3}^{\prime} \sin \varphi_{3}+l_{4} \sin \varphi_{4}+l_{5} \sin \varphi_{5}+l_{7} \sin \varphi_{7}=-y_{F_{0}} \\
& l_{3}^{\prime} \cos \varphi_{3}+l_{4} \cos \varphi_{4}+l_{5}^{\prime} \cos \varphi_{5}+l_{6} \cos \varphi_{6}=-x_{E_{0}} \\
& l_{3}^{\prime} \sin \varphi_{3}+l_{4} \sin \varphi_{4}+l_{5}^{\prime} \sin \varphi_{5}+l_{6} \sin \varphi_{6}=-y_{E_{0}} \\
& l_{7}^{\prime} \cos \left(\varphi_{7}-\alpha_{7}\right)-l_{8} \cos \varphi_{8}-s_{12}=0 \\
& l_{7}^{\prime} \sin \left(\varphi_{7}-\alpha_{7}\right)-l_{8} \sin \varphi_{8}=0 \\
& l_{6}^{\prime} \cos \varphi_{6}-l_{9} \cos \varphi_{9}+l_{10} \cos \varphi_{10}=-\left(x_{H_{0}}-x_{E_{0}}\right) \\
& l_{6}^{\prime} \sin \varphi_{6}-l_{9} \sin \varphi_{9}+l_{10} \sin \varphi_{10}=-\left(y_{H_{0}}-y_{E_{0}}\right) \\
& l_{10}^{\prime} \cos \left(\varphi_{10}+\alpha_{10}\right)-l_{11} \cos \varphi_{11}+s_{13}=0 \\
& l_{10}^{\prime} \sin \left(\varphi_{10}+\alpha_{10}\right)-l_{11} \sin \varphi_{11}=0
\end{aligned}
$$

This system with twelve scalar equations $(2,3, \ldots, 13)$ can be divided in five systems of nonlinear equations, of which four systems of two equations (specific to the dyad chain) and one system of four nonlinear equations (specific to the triad chain).

The resolving order of these systems of two or four nonlinear equations is given by the structural formula (1).

Using MathCAD software the kinematics of the bus door linkage has been accomplished. The software managed to resolve the twelve equation system only in stages as it has been mentioned above. The twelve variables (ten angular and two linear displacements) have been displayed in figure 7 and respectively in figure 8 . Along with these graphs the numerical tables 1 and 2 were also shown.

As it can be observed in figure 8, the linear displacements $s_{12}$ and $s_{13}$ have pretty matched trajectories which means that the mechanism is operating almost in a symmetry way. This is a positive sign regarding the working balance between the two left/right folding doors.

In figure 9 the angular and linear velocities have been displayed. Also, in figure 10 the angular and linear accelerations have been displayed. The issue of "how fast the bus door should operate?" is an optimization problem. If the speed of it is too high, the passengers could be struck, and if it is too low, the bus will be delayed. 
The main advantage of folding bus doors is the space saving inside the bus and the safety that it can provide to the passengers.

Table 1. Angular displacements of the mechanism elements
Table 2. Linear displacements of the mechanism elements

\begin{tabular}{|r|r|r|r|}
\hline 1 & \multicolumn{1}{|l|}{$\mathrm{S} 12$} & \multicolumn{1}{l|}{$\mathrm{S} 13$} & \multicolumn{1}{l|}{ s1k } \\
\hline 2 & 0.00000 & 0.00000 & 0 \\
\hline 3 & 0.003554 & 0.011774 & 0.01 \\
\hline 4 & 0.079337 & 0.101838 & 0.02 \\
\hline 5 & 0.151249 & 0.184608 & 0.03 \\
\hline 6 & 0.218171 & 0.258754 & 0.04 \\
\hline 7 & 0.279612 & 0.324069 & 0.05 \\
\hline 8 & 0.335495 & 0.380991 & 0.06 \\
\hline 9 & 0.385983 & 0.430254 & 0.07 \\
\hline 10 & 0.431359 & 0.472686 & 0.08 \\
\hline 11 & 0.471945 & 0.509081 & 0.09 \\
\hline 12 & 0.508045 & 0.540151 & 0.1 \\
\hline 13 & 0.539914 & 0.566489 & 0.11 \\
\hline 14 & 0.567727 & 0.588564 & 0.12 \\
\hline 15 & 0.591549 & 0.606712 & 0.13 \\
\hline 16 & 0.611291 & 0.621114 & 0.14 \\
\hline 17 & 0.626621 & 0.631757 & 0.15 \\
\hline 18 & 0.636757 & 0.638311 & 0.16 \\
\hline
\end{tabular}

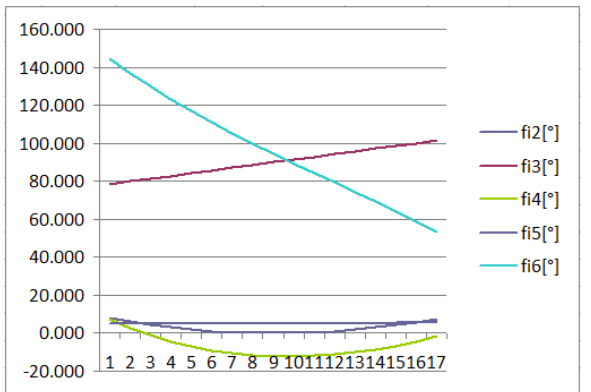

a)

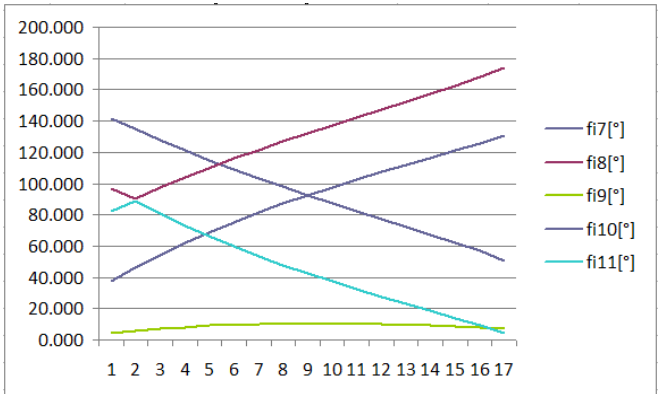

b)

Figure 7. Graphs of the angular displacements: $\varphi_{2}$ to $\varphi_{6}(a)$ and $\varphi_{7}$ to $\varphi_{11}(b)$

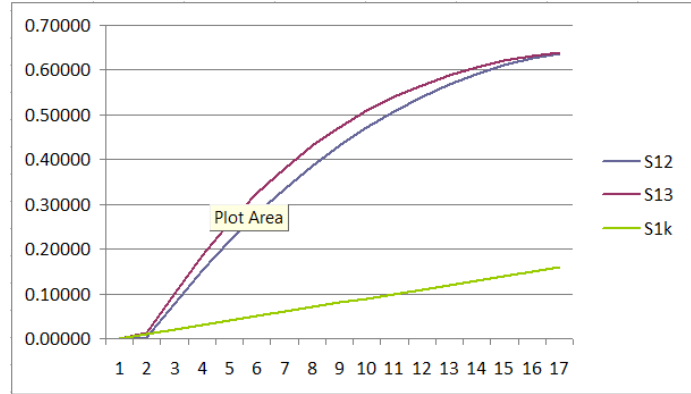

Figure 8. Graphs of the linear displacements: $s_{12}$ and $s_{13}$

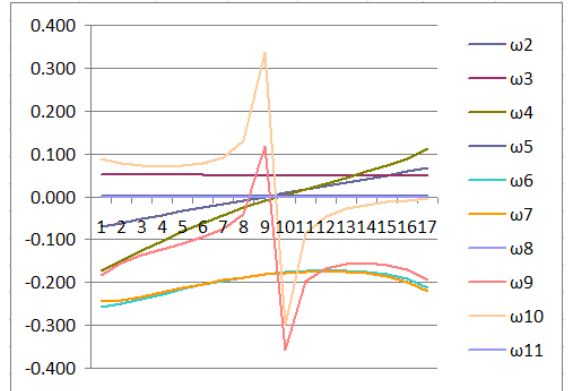

a)

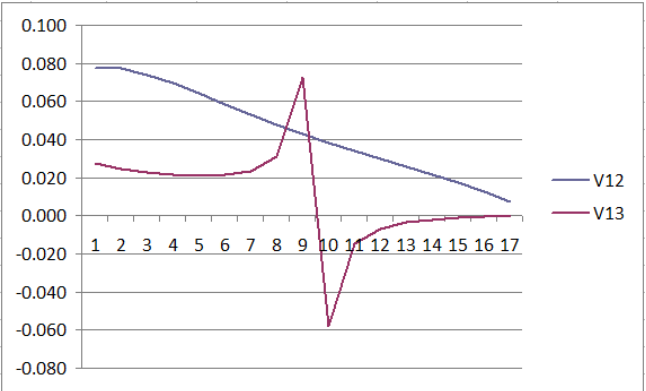

b)

Figure 9. Graphs of the angular/linear velocities: $\omega_{2}$ to $\omega_{11}(a), \mathrm{v}_{12}$ and $\mathrm{v}_{13}(b)$ 


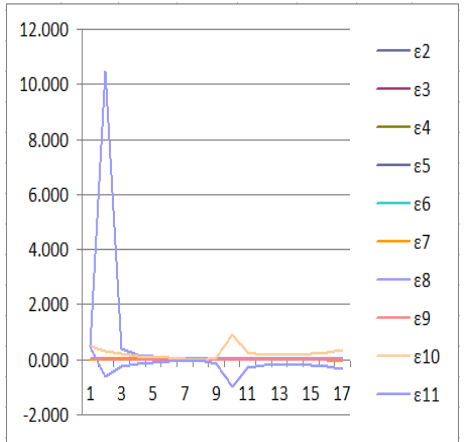

a)

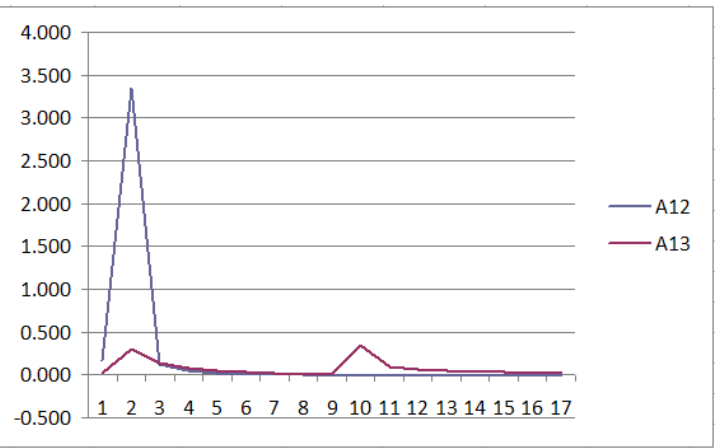

b)

Figure 10. Graphs of the angular/linear accelerations: $\varepsilon_{2}$ to $\varepsilon_{11}(a), \mathrm{a}_{12}$ and $\mathrm{a}_{13}(b)$

\section{Conclusions}

The paper presents a geometric-kinematic modeling of an articulated bar mechanism for actuate the doors of the urban buses.

It is emphasized that in the linear displacement graph of the kinematics of the linkage final elements, the two curves are nearly matched. This is pretty acceptable due to the fact that the mechanism should operate in a synchronized way.

Also, it has been outlined that the velocities and accelerations of the folding doors are very important for the passenger comfort and for the tight schedule of the bus driver. An optimized operation of the mechanism is the solution to this matter.

\section{References}

[1] Antonescu P 1970 Synthesis for relative-associated positions, the particular case of the positional synthesis (in Romanian), The 2-nd Conference on Technical Mechanics (vol. 11)

[2] Antonescu E Antonescu P and Fratila Gh 1972 Synthesis of the mechanisms for actuate the urban bus doors (in Romanian), Mechanism and Mechanical Transmission Symposium, Resita

[3] Antonescu D Veliscu V 2012 Analysis and synthesis of planar mechanisms used to generate curve line translation motion, Mechanism and Manipulator Journal, no. 2 (vol. 11)

[4] Copilus C Veliscu V 2014 An approach regarding windshield wiper mechanism design, SMAT International Congress, Craiova

[5] Hartenberg R Denavit D 1968 Kinematics Synthesis of Linkages, New-York

[6] Kovacs Fr. et al. 1971 On the synthesis of a function-generator mechanism on the basis of imposed precision conditions (in Romanian), Machine Design Journal, no. 12

[7] Lichtenheldt W 1970 Konstruktionslehre der Getriebe, Akad. Verlag, Berlin

[8] Manolescu N I Antonescu P 1970 Systemizing of the tri-positioning synthesis of the planar mechanisms with 4 elements of diverse design types (in Romanian), The 2-nd Conference on Technical Mechanics (vol. II)

[9] Manolescu N I et al. 1963 Exercise book of Mechanism and Machine Theory (in Romanian) (vol. I), Bucharest

[10] Manolescu N I Erceanu I Antonescu P 1962 Domain of Applicability of kinematic research of articulated planar mechanisms (in Romanian), S.C.M.A. no. 6

[11] Manolescu N I 1969 Contributions to numerical, structural and kinematic synthesis of Assur groups, of kinematic chains, of articulated planar mechanisms and "motor-mechanisms" (in Romanian), PhD Thesis

[12] Veliscu V Antonescu D Mesarici D 2015 Mechanisms used for driving windows of car side doors, 8th Symposium on Durability and Reliability of Mechanical Systems - SYMECH

[13] Veliscu V Mesarici D Antonescu P 2015 Topological structure and mobility of mechanisms used in car mechanical jacks, 8th Symposium on Durability and Reliability of Mechanical Systems, SYMECH

[14] Veliscu V Antonescu O Brezeanu C 2015 Main types of mechanisms used as windshield wipers, 8th Symposium on Durability and Reliability of Mechanical Systems, SYMECH

[15] www.autoline-eu.ro

[16] *** Technical documents of buses TV-20, Skoda, Ikarus-180 and trolleybus TV

[17] www.slideshare.net/WaleedAlyafie/automatic-door-of-bus-door 